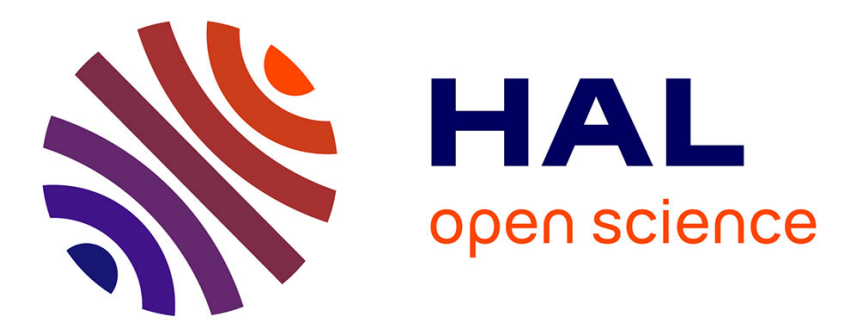

\title{
Invariant sets computation for convex difference inclusions systems
}

Mirko Fiacchini, Teodoro Alamo, Eduardo Camacho

\section{To cite this version:}

Mirko Fiacchini, Teodoro Alamo, Eduardo Camacho. Invariant sets computation for convex difference inclusions systems. Systems and Control Letters, 2012, 61 (8), pp.819-826. 10.1016/j.sysconle.2012.04.012 . hal-00984646

\section{HAL Id: hal-00984646 https://hal.science/hal-00984646}

Submitted on 5 Jul 2021

HAL is a multi-disciplinary open access archive for the deposit and dissemination of scientific research documents, whether they are published or not. The documents may come from teaching and research institutions in France or abroad, or from public or private research centers.
L'archive ouverte pluridisciplinaire HAL, est destinée au dépôt et à la diffusion de documents scientifiques de niveau recherche, publiés ou non, émanant des établissements d'enseignement et de recherche français ou étrangers, des laboratoires publics ou privés. 


\title{
Invariant Sets Computation for Convex Difference Inclusions Systems
}

\author{
M. Fiacchini ${ }^{\mathrm{a}}$, T. Alamo ${ }^{\text {, }}$, E.F. Camacho ${ }^{\mathrm{b}}$ \\ ${ }^{a}$ CRAN-ENSEM, 2 avenue de la foret de Haye, 54516 Vandoeuvre cedex, France. \\ ${ }^{b}$ Departamento de Ingeniería de Sistemas y Automática. Universidad de Sevilla. \\ Camino de los Descubrimientos s/n. 41092 Sevilla. Spain.
}

\begin{abstract}
In this paper we introduce the Convex Difference Inclusion (CDI) systems as a modelling framework useful to analyse set-theory and invariance-related issues for nonlinear and uncertain systems. The dynamics of a CDI system is given by a set-valued map whose values are convex, compact subsets of the space and are determined by convex bounding functions. Necessary and sufficient boundary-type conditions for invariance and contractiveness, characteristic of the linear systems, are given for the CDI systems. Lyapunov functions are proved to be induced by contractive sets for CDI systems, as in the linear context. A computational procedure for obtaining polytopic invariant and contractive sets for nonlinear systems, based on the properties of the CDI systems, is presented.
\end{abstract}

Keywords: Invariance, difference inclusions, convex analysis, nonlinear systems.

\section{Introduction}

Invariance and contractiveness are fundamental in systems analysis and control, mainly due to the stability and robustness properties of these regions of the state space. A notable pioneering contribution on invariance is [1]. Invariance and related topics, mainly for linear systems, are treated in $[2,3,4,5]$, on the maximal invariant set, and in [6], on the minimal invariant set. A monograph on the topic is [7]. Invariance is also employed to ensure convergence of model predictive control, see $[8,9]$. Few general results are available for nonlinear systems.

Email addresses: mirko.fiacchini@ensem.inpl-nancy.fr(M. Fiacchini), alamo@cartuja.us.es (T. Alamo), eduardo@cartuja.us.es (E.F. Camacho) 
The problems of obtaining invariant ellipsoids, [10], and parallelotopes, [11], for nonlinear systems, are addressed using linear difference inclusions (LDI). The computation of invariant ellipsoids for linear systems with static nonlinear functions in the feedback, as piecewise affine functions and saturation, are addressed in $[12,13]$. Methods to obtain invariant polytopes are proposed for saturated systems, [14] and for Lur'e systems, [15]. The computation of invariant polytopes for general nonlinear systems is discussed in [16], using interval arithmetic, and in [17, 18], employing properties of DC functions. The work [19] proposes approximations of the minimal invariant set for quantized systems.

In this paper we present and use CDI systems for representing and approximating nonlinear and uncertain discrete-time systems. The CDI systems are tightly related to differential and difference inclusions. See [20, 21, 22] for a deep and exhaustive analysis of such models and of their properties. Nevertheless, and despite their generality and mathematical rigor, the impression is that the results of the cited works have still not found the central role they deserve, mainly in the more practical and computation-oriented fields of control.

Our aim is to particularize the analysis posing convexity-related assumptions on the set-valued maps and on the considered sets. This implies less generality but it also permits to exploit the properties of difference inclusions and convex analysis (see [23, 24, 25]), for computing invariant and contractive sets for nonlinear and uncertain systems. From another point of view, CDI systems are the result of an abstraction process to generalize previous results for particular nonlinear systems, see [18] for instance. Necessary and sufficient boundary-type conditions for invariance and contractiveness of convex sets for CDI systems are stated. Such results are employed to design an algorithm to obtain invariant and contractive polytopes for CDI systems. Since many nonlinear systems admit CDI representations or extensions, the results apply to a wide class of systems.

The paper is organized as follows: Section II introduces the CDI systems. Section III presents invariance and contractiveness for CDI systems. In Section IV the algorithm is illustrated and then applied to a numerical example in Section $\mathrm{V}$. The paper ends with a section of conclusions.

Notation: The set of positive integers smaller than or equal to $n \in \mathbb{N}$ is $\mathbb{N}_{n}$. Given $A \in \mathbb{R}^{n \times m}, A_{i}$ with $i \in \mathbb{N}_{n}$, is its $i$-th row. Given a set $D \subseteq \mathbb{R}^{n}, \operatorname{co}(D)$ is the convex hull of $D, \operatorname{int}(D)$ its interior, $\partial D$ its boundary, $\mathcal{S}(D)$ are the subsets of $D, \mathcal{K}(D)$ are the convex compact subsets of $D$ and $\mathcal{K}^{0}(D)$ are the convex compact sets $C \subseteq D$ with $0 \in \operatorname{int}(C)$. Given $D, E \subseteq \mathbb{R}^{n}$ and $\alpha \geq 0$, define $D \oplus E=\{z=$ $\left.x+y \in \mathbb{R}^{n}: x \in D, y \in E\right\}$ and $\alpha D=\left\{\alpha x \in \mathbb{R}^{n}: x \in D\right\}$. Given a set-valued $\operatorname{map} F: \mathbb{R}^{n} \rightarrow \mathcal{S}\left(\mathbb{R}^{m}\right)$, define $\operatorname{graph}(F)=\left\{(x, y) \in \mathbb{R}^{n} \times \mathbb{R}^{m}: y \in F(x)\right\}$. 


\section{Convex difference inclusions: CDI systems}

Consider the system given by the difference inclusions

$$
x^{+} \in \mathcal{F}(x),
$$

where $x \in \mathbb{R}^{n}$ is the state, $x^{+}$is the successor and $\mathcal{F}: \mathbb{R}^{n} \rightarrow \mathcal{S}\left(\mathbb{R}^{n}\right)$ is a set-valued map on $\mathbb{R}^{n}$, that is a function which relates a set to every point $x \in \mathbb{R}^{n}$.

Assumption 1. The set-valued map $\mathcal{F}: \mathbb{R}^{n} \rightarrow \mathcal{K}\left(\mathbb{R}^{n}\right)$ determining the system dynamics (1) is such that

$$
\mathcal{F}\left(\alpha x^{1}+(1-\alpha) x^{2}\right) \subseteq \alpha \mathcal{F}\left(x^{1}\right) \oplus(1-\alpha) \mathcal{F}\left(x^{2}\right),
$$

for every $\alpha \in[0,1]$ and every $x^{1}, x^{2} \in \mathbb{R}^{n}$, and $\mathcal{F}(0)=\{0\}$.

Notice that Assumption 1 implies also that $\mathcal{F}(x)$ is convex and compact for every $x \in \mathbb{R}^{n}$. The dynamical systems (1) for which Assumption 1 holds are referred to as Convex Difference Inclusions (CDI) systems. Consider the system

$$
x^{+} \in \mathcal{F}(x) \oplus W
$$

where $x \in \mathbb{R}^{n}$ is the state, $x^{+}$is the successor, $\mathcal{F}(\cdot)$ is a set-valued map on $\mathbb{R}^{n}$ and $W$ is the additive uncertainty bounding set satisfying the following assumption:

Assumption 2. The set $W \subseteq \mathbb{R}^{n}$ is compact and $0 \in \operatorname{int}(\operatorname{co}(W))$.

If Assumptions 1 and 2 hold for $\mathcal{F}(\cdot)$ in (3) the system is denoted as uncertain CDI system. We recall here the concept of support function.

Definition 1. Given a set $D \subseteq \mathbb{R}^{n}$, the support function of $D$ evaluated at $\eta \in \mathbb{R}^{n}$ is $\phi_{D}(\eta)=\sup _{x \in D} \eta^{T} x$.

Among the properties of support function, see [23, 24], we have that set inclusion conditions can be given in terms of support functions.

Property 1. Given a closed, convex set $D \subseteq \mathbb{R}^{n}$, then $x \in D$ if and only if $\eta^{T} x \leq \phi_{D}(\eta)$, for all $\eta \in \mathbb{R}^{n}$. Given also $C \subseteq \mathbb{R}^{n}$, then $C \subseteq D$ if and only if $\phi_{C}(\eta) \leq \phi_{D}(\eta)$, for all $\eta \in \mathbb{R}^{n}$.

Assumption 1 can be posed also in terms of support functions, see below. 
Proposition 1. The set-valued map $\mathcal{F}: \mathbb{R}^{n} \rightarrow \mathcal{K}\left(\mathbb{R}^{n}\right)$ determining the system dynamics (1) satisfies Assumption 1 if and only if $\check{F}: \mathbb{R}^{n} \times \mathbb{R}^{n} \rightarrow \mathbb{R}$ defined as

$$
\check{F}(x, \eta)=\sup _{z \in \mathcal{F}(x)} \eta^{T} z
$$

is such that $\check{F}(\cdot, \eta)$ is convex on $\mathbb{R}^{n}$ and $\check{F}(0, \eta)=0$, for all $\eta \in \mathbb{R}^{n}$.

Proof: The proposition, suggested to us by a reviewer we would like to acknowledge, stems from properties of support functions. Notice that the value $\check{F}(x, \eta)$ is the support function at $\eta \in \mathbb{R}^{n}$ of the set $\mathcal{F}(x)$, for every $x \in \mathbb{R}^{n}$. From $\mathcal{F}(x) \in \mathcal{K}\left(\mathbb{R}^{n}\right)$ and properties of support function, see [23, 24], the relation (2) holds for every $\eta \in \mathbb{R}^{n}$, every $\alpha \in[0,1]$ and every $x^{1}, x^{2} \in \mathbb{R}^{n}$, if and only if

$$
\begin{aligned}
& \check{F}\left(\alpha x^{1}+(1-\alpha) x^{2}, \eta\right)=\phi_{\mathcal{F}\left(\alpha x^{1}+(1-\alpha) x^{2}\right)}(\eta) \leq \phi_{\alpha \mathcal{F}\left(x^{1}\right) \oplus(1-\alpha) \mathcal{F}\left(x^{2}\right)}(\eta)= \\
& \quad=\alpha \phi_{\mathcal{F}\left(x^{1}\right)}(\eta)+(1-\alpha) \phi_{\mathcal{F}\left(x^{2}\right)}(\eta)=\alpha \check{F}\left(x^{1}, \eta\right)+(1-\alpha) \check{F}\left(x^{2}, \eta\right)
\end{aligned}
$$

which means that $\check{F}(x, \eta)$ is convex in $x$, for every $\eta \in \mathbb{R}^{n}$. Finally, $\mathcal{F}(0)=\{0\}$ if and only if $\check{F}(0, \eta)=0$ for all $\eta \in \mathbb{R}^{n}$.

The function $\check{F}(\cdot, \cdot)$ is referred to as convex bounding function.

Remark 1. The function $\check{F}(\cdot, \eta)$ is continuous on the relative interior of its effective domain, for every $\eta \in \mathbb{R}^{n}$, from its convexity, see Th.10.1 in [23]. This and the fact that $\mathcal{F}(x)$ is assumed convex and compact for every $x \in \mathbb{R}^{n}$ imply that $\mathcal{F}$ is continuous on $\mathbb{R}^{n}$ and is a particular case of Marchaud maps, often considered in works concerning viability theory and set-valued dynamical systems, [20, 21, 22].

By convexity and compactness of $\mathcal{F}(x)$ for every $x \in \mathbb{R}^{n}$, we have that

$$
\mathcal{F}(x)=\left\{z \in \mathbb{R}^{n}: \eta^{T} z \leq \check{F}(x, \eta), \forall \eta \in \mathbb{R}^{n}\right\} .
$$

Given two set-valued maps $G, F: \mathbb{R}^{n} \rightarrow \mathcal{S}\left(\mathbb{R}^{n}\right)$, we say that $G$ is an extension of $F$, and write $F \subseteq G$, if and only if $\operatorname{graph}(F) \subseteq \operatorname{graph}(G)$. A system is an extension of another if the graph of the former is an extension of the graph of the latter. The CDI systems contain a large class of nonlinear and uncertain systems and can be used to approximate many others, see Proposition 2 below and [26].

Proposition 2. Consider the system $x^{+}=f(x)$ with $f: \mathbb{R}^{n} \rightarrow \mathbb{R}^{n}$ twice differentiable in $D=\left\{x \in \mathbb{R}^{n}:\left\|x-x_{0}\right\|_{2}<r\right\}$, with $r>0$, and $\rho \in \mathbb{R}^{n}$ such that

$$
\left|\frac{1}{2}\left(x-x_{0}\right)^{T} H^{j}(\tilde{x})\left(x-x_{0}\right)\right| \leq \rho_{j}\left(x-x_{0}\right)^{T}\left(x-x_{0}\right),
$$


for all $x, \tilde{x} \in D$, with $j \in \mathbb{N}_{n}$, where $H\left(f_{j}\right)(\cdot)=H^{j}(\cdot)$, is the Hessian of $f_{j}(\cdot)$. Then the CDI system defined by (5) with the convex bounding functions

$\check{F}\left(x-x_{0}, \eta\right)=\sum_{j=1}^{n}\left\{\eta_{j}\left(f_{j}\left(x_{0}\right)+\left(x-x_{0}\right)^{T} \nabla f_{j}\left(x_{0}\right)\right)+\rho_{j}\left|\eta_{j}\right|\left(x-x_{0}\right)^{T}\left(x-x_{0}\right)\right\}$,

for every $\eta \in \mathbb{R}^{n}$, is an extension of the nonlinear one, on $D$.

Proof: By hypothesis, the gradient $\nabla f_{j}(\cdot)$ and the Hessian of $f_{j}(\cdot)$ exist at every $x \in D$, for all $j \in \mathbb{N}_{n}$. Exploiting the Lagrange form of the remainders of the Taylor series expansion, we have that given $x_{0} \in D$, for every $x \in D$ there exists $\tilde{x}(x)=\tilde{x} \in D$ such that the following equality holds

$$
f_{j}(x)=f_{j}\left(x_{0}\right)+\left(x-x_{0}\right)^{T} \nabla f_{j}\left(x_{0}\right)+\frac{1}{2}\left(x-x_{0}\right)^{T} H^{j}(\tilde{x})\left(x-x_{0}\right),
$$

for every $j \in \mathbb{N}_{n}$. From (6), for all $x \in D$ and every $\eta \in \mathbb{R}^{n}$, we have that

$$
\begin{aligned}
& \eta^{T} f(x)=\sum_{j=1}^{n} \eta_{j}\left(f_{j}\left(x_{0}\right)+\left(x-x_{0}\right)^{T} \nabla f_{j}\left(x_{0}\right)+\frac{1}{2}\left(x-x_{0}\right)^{T} H^{j}\left(\tilde{x}^{j}\right)\left(x-x_{0}\right)\right) \leq \\
\leq & \sum_{j=1}^{n} \eta_{j}\left(f_{j}\left(x_{0}\right)+\left(x-x_{0}\right)^{T} \nabla f_{j}\left(x_{0}\right)\right)+\left|\eta_{j}\right|\left|\frac{1}{2}\left(x-x_{0}\right)^{T} H^{j}\left(\tilde{x}^{j}\right)\left(x-x_{0}\right)\right| \leq \check{F}\left(x-x_{0}, \eta\right)
\end{aligned}
$$

which means that $f \subseteq \mathcal{F}$, where $\mathcal{F}(\cdot)$ is defined by (5) and (7).

A possible choice of $\rho_{j}$ is the maximum on $D$ of the spectral norm of $0.5 H^{j}(\tilde{x})$.

Remark 2. For a nonlinear system $x^{+}=f(x)$, a finite number of convex bounding functions can be sufficient to determine a CDI extension. For instance, if for every $i \in \mathbb{N}_{n}$ there exist two functions $\check{f}_{i}, \hat{f}_{i}: \mathbb{R}^{n} \rightarrow \mathbb{R}$, convex and concave, respectively, such that $\hat{f}_{i}(x) \leq f_{i}(x) \leq \check{f}_{i}(x)$, for all $x \in \mathbb{R}^{n}$, and $\check{f}_{i}(0)=\hat{f}_{i}(0)=$ 0 , then a CDI system extension of the nonlinear one can be determined.

The convexity of the bounding functions, implied by Assumption 1 (see Proposition 1), permits to characterize invariant sets in terms of convex constraints and then to pose efficiently solvable problems for their computation, see [25].

\subsection{LDI systems}

A popular way of approximating nonlinear and uncertain systems is given by Linear Difference Inclusion (LDI) systems, see [27, 28]. It will be shown that the LDI systems form a subclass of the CDI ones, in particular of those whose 
convex bounding functions are piecewise linear. Hence, using an LDI system to approximate a nonlinear one is a way of generating a CDI extension.

An LDI system in terms of difference inclusions is given by (1) with

$$
\mathcal{F}(x)=\mathcal{A}(x)=\left\{A x \in \mathbb{R}^{n}: A \in \mathcal{A}\right\},
$$

where, with a slight abuse of notation, we use $\mathcal{A}$ for denoting both the set-valued map and the set $\mathcal{A} \subseteq \mathbb{R}^{n \times n}$. If $\mathcal{A}$ is a polytope in $\mathbb{R}^{n \times n}$, the LDI is said polytopic.

Remark 3. Notice that the set-valued map $\mathcal{A}(\cdot)$ satisfies the Assumption 1 if $\mathcal{A}(x) \in \mathcal{K}\left(\mathbb{R}^{n}\right)$ for all $x \in \mathbb{R}^{n}$ (then also polytopic LDIs do). In fact, the function

$$
\check{F}(x, \eta)=\sup _{z \in \mathcal{A}(x)} \eta^{T} z=\max _{A \in \mathcal{A}} \eta^{T} A x
$$

with $\eta \in \mathbb{R}^{n}$, is convex in $x$, being the pointwise maximum of a family of convex functions, see [25]. Moreover, $\check{F}(0, \eta)=\{0\}$ for all $\eta \in \mathbb{R}^{n}$. Then the LDI systems are a particular subclass of the CDI systems and hence every result valid for the latters applies also to the formers. Nonetheless CDI provides a more general modelling framework, as not every CDI system admits an LDI representation.

Remark 4. Important results, valid for linear systems, are valid also for LDI systems (more generally, for positively homogeneous ones). An example is the boundary-type condition for invariance and contractiveness, see Sec. 4.2.4 in [7]. The underlying idea is that, if the extremal realizations of the LDI, which are linear systems, satisfy a condition (invariance for instance), then the whole LDI system fulfils it, see [27, 28, 29]. Such results are substantially based on linearity. The key idea of the CDI approach is that the fundamental ingredient for the desired invariance-related properties to hold is convexity rather than linearity. Thus the results for the CDI systems improve and contain those for the LDI ones.

\subsection{Generalized saturated systems}

Generalized saturated systems, introduced in [30], are a family of systems including many common static nonlinearities (saturation, dead-zone, hysteresis, etc.) and are easily extendible by CDI systems. We introduce the definition of generalized saturated function in its scalar version (see [30] for the vectorial one).

Definition 2. The function $\varphi: \mathbb{R} \times \mathbb{N} \rightarrow \mathbb{R}$ is said to be a generalized saturated function with saturation level $y_{0} \in \mathbb{R}, y_{0}>0$, dead-zone $\sigma \in \mathbb{R}^{n}, \sigma \geq 0$, and linear slope $\mu \in \mathbb{R}, \mu>0$, if

$$
-\Gamma(-y) \leq \varphi(y, k) \leq \Gamma(y), \quad \forall y \in \mathbb{R}, \forall k \in \mathbb{N},
$$

where $\Gamma(y)=\max \left\{\mu(y+\sigma),-y_{0}\right\}$ and $k \in \mathbb{N}$ is the discrete-time instant. 
The generalized saturated functions can represent common static nonlinear functions as saturation plus dead-zone, hysteresis (see Figure 1) and saturation.
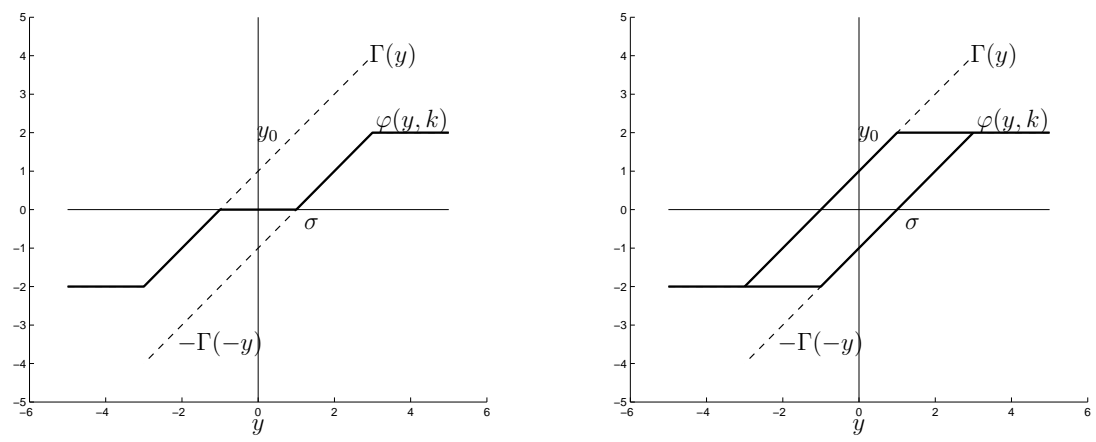

Figure 1: Examples: saturation plus dead-zone (left) and hysteresis (right).

Given the generalized saturated function $\varphi(\cdot, \cdot)$, the dynamical system

$$
x_{k+1}=A x_{k}+B \varphi\left(F x_{k}, k\right),
$$

where $F \in \mathbb{R}^{1 \times n}$, is called generalized saturated system. A CDI extension of the generalized saturated system can be directly determined by the following convex bounding functions

$$
\check{F}(x, \eta)= \begin{cases}\eta^{T} A x+\eta^{T} B \Gamma^{0}(F x), & \text { if } \eta^{T} B \geq 0, \\ \eta^{T} A x-\eta^{T} B \Gamma^{0}(-F x), & \text { if } \eta^{T} B<0,\end{cases}
$$

for all $\eta \in \mathbb{R}^{n}$ and all $x \in \mathbb{R}^{n}$ with $\Gamma^{0}(y)=\max \left\{\mu y,-y_{0}-\mu \sigma\right\}$. The system (3) with $\mathcal{F}(\cdot)$ determined by convex bounding functions (10) and $W=\{w=B v$ : $-\mu \sigma \leq v \leq \mu \sigma\}$, is an uncertain CDI extension of the generalized saturated one.

Remark 5. Notice that the generalized saturated systems do not admit LDI extensions. Even for simple saturated systems, the LDI extension is more conservative than the CDI one. In fact, given $\sigma=0$, the graph of the LDI approximation of the saturated system is obtained by replacing $\Gamma^{0}(y)$ with $\max \{\mu y, 0\}$ in (10). Thus the graph of the CDI extension is strictly contained in the graph of the LDI one.

\section{Invariance for CDI systems}

Invariance and contractiveness of convex sets for CDI systems are characterized in this section. First, the standard definitions are recalled. 
Definition 3. A set $\Omega \subseteq \mathbb{R}^{n}$ is a robust invariant set for the system $x^{+}=f(x, w)$ and constraints $x \in X$ if $\Omega \subseteq X$ and $f(x, w) \in \Omega$, for all $x \in \Omega$ and all $w \in W$.

In the absence of the uncertainty the related set is called invariant set.

Definition 4. A set $\Omega \in \mathcal{K}^{0}\left(\mathbb{R}^{n}\right)$ is a contractive set for the system $x^{+}=f(x, w)$ and constraints $x \in X$, with contracting factor $\lambda \in[0,1]$, if $\Omega \subseteq X$ and $f(x, w) \in \lambda \Omega$, for all $x \in \Omega$ and all $w \in W$.

Notice that contractiveness induces invariance, thus when in the following we will guarantee contractiveness of a set, we will implicitly ensure also invariance. In what follows we prove that important results valid for linear systems, concerning boundary-type conditions for invariance and set-induced Lyapunov functions, are valid also for CDI systems.

\subsection{Necessary and sufficient condition for invariance for CDI systems}

As invariance and set-theory are important to deal with control in presence of constraints, consider the state constraints $x \in X \subseteq \mathbb{R}^{n}$. The unconstrained case is enclosed, given by $X=\mathbb{R}^{n}$.

Assumption 3. The state constraint set $X \subseteq \mathbb{R}^{n}$ is closed, convex and $0 \in$ $\operatorname{int}(X)$.

A necessary and sufficient condition for contractiveness for CDI systems is provided, see [5] for the linear case. Given the set-valued map $\mathcal{F}(\cdot)$, define the $\operatorname{map} \mathcal{M}_{\mathcal{F}}: \mathcal{S}\left(\mathbb{R}^{n}\right) \rightarrow \mathcal{S}\left(\mathbb{R}^{n}\right)$ as

$$
\mathcal{M}_{\mathcal{F}}(\Omega)=\bigcup_{x \in \Omega} \mathcal{F}(x)
$$

for all $\Omega \in \mathcal{S}\left(\mathbb{R}^{n}\right)$, which is monotone, i.e. $\mathcal{M}_{\mathcal{F}}(C) \subseteq \mathcal{M}_{\mathcal{F}}(D)$ for all $C, D \subseteq \mathbb{R}^{n}$ such that $C \subseteq D$. Given a set $X_{0} \in \mathcal{S}\left(\mathbb{R}^{n}\right)$, the sequence of sets $X_{k}$, for $k \in \mathbb{N}$, generated by iterating

$$
X_{k+1}=\mathcal{M}_{\mathcal{F}}\left(X_{k}\right),
$$

with initial condition $X_{0}$ are the sets reachable from $x \in X_{0}$.

Property 2. The condition for contractiveness of a set $\Omega \in \mathcal{K}^{0}(X)$ for CDI systems is $\mathcal{F}(x) \subseteq \lambda \Omega$ for every $x \in \Omega$ or, equivalently, $\mathcal{M}_{\mathcal{F}}(\Omega) \subseteq \lambda \Omega$, where $\mathcal{M}_{\mathcal{F}}(\cdot)$ is defined in (11). 
The contractiveness of $\Omega \in \mathcal{K}^{0}(X)$ for a CDI system in terms of support functions follows.

Proposition 3. Let Assumptions 1 and 3 hold for the set-valued map $\mathcal{F}(\cdot)$ determining the system dynamics (1) and the state constraint set $X$. Given $\lambda \in[0,1]$, a set $\Omega \in \mathcal{K}^{0}(X)$ is a contractive set for system (1) if and only if

$$
\eta^{T} z \leq \lambda \phi_{\Omega}(\eta), \quad \forall z \in \mathcal{F}(x), \quad \forall x \in \Omega, \quad \forall \eta \in \mathbb{R}^{n}
$$

Proof: The condition for contractiveness can be expressed in terms of support functions as $\phi_{\mathcal{F}(x)}(\eta) \leq \lambda \phi_{\Omega}(\eta)$, for all $x \in \Omega$ and $\eta \in \mathbb{R}^{n}$, see Property 1 , then also as in (13).

Condition (13) involves every $x \in \Omega$. A boundary-type necessary and sufficient condition for contractiveness for CDI systems and convex sets can be posed. Given $\Omega \in \mathcal{K}^{0}\left(\mathbb{R}^{n}\right)$, the Minkowski function of $\Omega$ at $x \in \mathbb{R}^{n}$ is defined as

$$
\Psi_{\Omega}(x)=\min _{\alpha \geq 0}\{\alpha \in \mathbb{R}: x \in \alpha \Omega\}
$$

Theorem 1. Let Assumptions 1 and 3 hold for the set-valued map $\mathcal{F}(\cdot)$ determining the system dynamics (1) and the state constraint set $X$. Given $\lambda \in[0,1]$, a set $\Omega \in \mathcal{K}^{0}(X)$ is a contractive set for system (1) if and only if

$$
\check{F}(x, \eta) \leq \lambda \phi_{\Omega}(\eta), \quad \forall x \in \partial \Omega, \quad \forall \eta \in \mathbb{R}^{n} .
$$

Proof: Condition (14) is equivalent to $\mathcal{F}(x) \subseteq \lambda \Omega$ for $x$ on the boundary of $\Omega$. We prove that $\mathcal{F}(x) \subseteq \lambda \Omega$ is satisfied for every $x \in \partial \Omega$ if and only if it is satisfied for every $x \in \Omega$. Necessity is due to $\partial \Omega \subseteq \Omega$, since $\Omega$ is compact. To prove sufficiency, consider $x \in \Omega$. Then $\bar{x}=\alpha^{-1} x$, with $\alpha=\Psi_{\Omega}(x) \in[0,1]$, is such that $\bar{x} \in \partial \Omega$ and $x$ is the convex combination of the origin and $\bar{x}$, that is $x=\alpha \bar{x}+(1-\alpha) 0$. Assume that $\mathcal{F}(\bar{x}) \subseteq \lambda \Omega$ for all $\bar{x} \in \partial \Omega$, as implied by (14) and notice that, from Assumption 1, we have $\mathcal{F}(0)=\{0\} \subseteq \lambda \Omega$. From this and Assumption 1 we have that $\mathcal{F}(x)=\mathcal{F}(\alpha \bar{x}+(1-\alpha) 0) \subseteq \alpha \mathcal{F}(\bar{x}) \subseteq \alpha \lambda \Omega \subseteq \lambda \Omega$, and then $\mathcal{F}(x) \subseteq \lambda \Omega$ for all $\alpha \in \Omega$.

Theorem 1 provides a necessary and sufficient condition for contractiveness of $\Omega \in \mathcal{K}^{0}(X)$ for CDI systems, based on convex constraints concerning only the boundary of set $\Omega$. In general, the conditions for contractiveness for nonlinear systems involve every $x$ in $\Omega$, see [7]. The following propositions present the relation between contractive sets and Lyapunov stability theory for CDI systems. 
Proposition 4. Let Assumptions 1 and 3 hold for the set-valued map $\mathcal{F}(\cdot)$ determining the system dynamics (1) and the state constraint set $X$. For every contractive set $\Omega \in \mathcal{K}^{0}(X)$ for system (1) with contracting factor $\lambda \in[0,1]$, also the set $\alpha \Omega \subseteq X$, with $\alpha \in[0,1]$, is a convex, compact, contractive set for system (1) with contracting factor $\lambda$.

Proof: Compactness and convexity of $\alpha \Omega$ for all $\alpha \in[0,1]$ follow by definition. Suppose that $\mathcal{M}_{\mathcal{F}}(\Omega) \subseteq \lambda \Omega$ and consider $\alpha \in[0,1]$. By definition, $x \in \alpha \Omega$ is equivalent to the existence of $y \in \Omega$ such that $x=\alpha y$. Then, from Assumption 1 , we have

$$
\mathcal{M}_{\mathcal{F}}(\alpha \Omega)=\bigcup_{x \in \alpha \Omega} \mathcal{F}(x)=\bigcup_{y \in \Omega} \mathcal{F}(\alpha y) \subseteq \bigcup_{y \in \Omega} \alpha \mathcal{F}(y) \subseteq \bigcup_{y \in \Omega} \alpha \lambda \Omega=\alpha \lambda \Omega
$$

which means that $\alpha \Omega$ is a contractive set with contracting factor $\lambda$.

Proposition 4 implies that every contractive set for a CDI system induces a local Lyapunov function, as shown below. Analogous results are valid for linear and particular nonlinear systems, see [7].

Definition 5. Given $\Omega \in \mathcal{K}^{0}(X)$, the function $\mathcal{V}_{\Omega}: \mathcal{S}(X) \rightarrow \mathbb{R}$ defined as

$$
\mathcal{V}_{\Omega}(D)=\sup _{x \in D} \Psi_{\Omega}(x)=\min _{\alpha \geq 0}\{\alpha \in \mathbb{R}: D \subseteq \alpha \Omega\}
$$

is a local Lyapunov function in $\mathcal{S}(X)$ for the CDI system (1), if $\mathcal{V}_{\Omega}\left(\mathcal{M}_{\mathcal{F}}(D)\right)<$ $\mathcal{V}_{\Omega}(D)$ for every $D \in \mathcal{S}(X) \backslash\{0\}$.

Notice in fact that a function $\mathcal{V}_{\Omega}(\cdot)$ as in Definition 5 is positive definite in $\mathcal{S}(X), \mathcal{V}_{\Omega}(D)=0$ if and only if $D=\{0\}$ and it decreases along the set-valued trajectory generated by (12) with $X_{0} \in \mathcal{S}(X) \backslash\{0\}$.

Proposition 5. Let Assumptions 1 and 3 hold for the set-valued map $\mathcal{F}(\cdot)$ determining the system (1). The function $\mathcal{V}_{\Omega}(\cdot)$ defined as in (15) is a local Lyapunov function in $\mathcal{S}(\Omega)$ for the system (1), for every contractive set $\Omega \in \mathcal{K}^{0}(X)$ with contracting factor $\lambda \in[0,1)$.

Proof: Consider $D \in \mathcal{S}(\Omega)$ such that $\mathcal{V}_{\Omega}(D)=\alpha$ with $\alpha \in(0,1]$, then $D \subseteq \alpha \Omega \subseteq \Omega$. From monotonicity of $\mathcal{M}_{\mathcal{F}}(\cdot)$ and Proposition 4, it follows that $\mathcal{M}_{\mathcal{F}}(D) \subseteq \mathcal{M}_{\mathcal{F}}(\alpha \Omega) \subseteq \lambda \alpha \Omega$, with $\alpha \in(0,1]$, which implies

$$
\mathcal{V}_{\Omega}\left(\mathcal{M}_{\mathcal{F}}(D)\right) \leq \mathcal{V}_{\Omega}\left(\mathcal{M}_{\mathcal{F}}(\alpha \Omega)\right) \leq \mathcal{V}_{\Omega}(\lambda \alpha \Omega)=\lambda \alpha<\alpha=\mathcal{V}_{\Omega}(D)
$$


since $\mathcal{V}_{\Omega}(\beta \Omega)=\beta$, for all $\beta \geq 0$, and $\mathcal{V}_{\Omega}(C) \leq \mathcal{V}_{\Omega}(E)$ for all $C, E \in \mathcal{S}\left(\mathbb{R}^{n}\right)$ such that $C \subseteq E$. If $\alpha=0$, then $D=\{0\}$ and the inequalities in (16) become equalities. Hence, $\mathcal{V}_{\Omega}\left(\mathcal{M}_{\mathcal{F}}(D)\right)<\mathcal{V}_{\Omega}(D)$, for all $D \in \mathcal{S}(\Omega) \backslash\{0\}$.

Proposition 5 implies that $\lambda \in[0,1)$ is a bound on the decreasing rate of the Lyapunov function along the trajectories. That is, given $X_{0} \in \mathcal{S}(\Omega)$ (with $X_{0} \neq$ $\{0\})$, we have that $\mathcal{V}_{\Omega}\left(X_{k+1}\right) \leq \lambda \mathcal{V}_{\Omega}\left(X_{k}\right)<\mathcal{V}_{\Omega}\left(X_{k}\right)$, and then $\mathcal{V}_{\Omega}\left(X_{k}\right) \leq \lambda^{k}$, for all $k \in \mathbb{N}$. Geometrically, it means that $X_{0} \subseteq \Omega$ implies $X_{k} \subseteq \lambda^{k} \Omega$ for all $k \in \mathbb{N}$. Hence given $X_{0} \in \mathcal{S}(\Omega)$ as initial condition, the set-valued trajectory converges to the set composed by the origin and the system is exponentially stable.

Proposition 6. Let Assumptions 1 and 3 hold for the set-valued map $\mathcal{F}(\cdot)$ determining the system (1). Given two contractive sets $\Lambda \in \mathcal{K}^{0}(X)$ and $\Gamma \in \mathcal{K}^{0}(X)$ for the system (1) with contracting factors $\lambda \in[0,1]$ and $\gamma \in[0,1]$, respectively, the set $\Omega=\operatorname{co}(\Lambda, \Gamma) \in \mathcal{K}^{0}(X)$ is a contractive set with contracting factor $\omega=\max \{\lambda, \gamma\}$.

Proof: Compactness and convexity of $\Omega$ and $0 \in \operatorname{int}(\Omega)$ follow by definition of convex hull. Moreover $\Omega \subseteq X$ since $X$ is convex, $\Lambda \subseteq X$ and $\Gamma \subseteq X$, which implies that any convex combination of elements of $\Lambda$ and $\Gamma$ belongs to $X$. Suppose that $\mathcal{F}(x) \subseteq \lambda \Lambda$ for all $x \in \Lambda$ and $\mathcal{F}(x) \subseteq \gamma \Gamma$ for all $x \in \Gamma$. For every $x \in \operatorname{co}(\Lambda, \Gamma)=\Omega$, there exist $y \in \Lambda, z \in \Gamma$ and $\alpha \in[0,1]$ such that $x=\alpha y+(1-\alpha) z$. Then, from Assumption 1 and convexity of $\Gamma$ and $\Lambda$, and properties of convex sets, see [23, 24], we have

$$
\begin{aligned}
& \mathcal{F}(x)=\mathcal{F}(\alpha y+(1-\alpha) z) \subseteq \alpha \mathcal{F}(y) \oplus(1-\alpha) \mathcal{F}(z) \subseteq \alpha \lambda \Lambda \oplus(1-\alpha) \gamma \Gamma \subseteq \\
& \subseteq \alpha \lambda \Omega \oplus(1-\alpha) \gamma \Omega=(\alpha \lambda+(1-\alpha) \gamma) \Omega \subseteq(\alpha \omega+(1-\alpha) \omega) \Omega=\omega \Omega,
\end{aligned}
$$

for every $x \in \Omega$. Then $\Omega$ is contractive with contracting factor $\omega$.

The following corollary shows that no loss of generality is induced by assuming convexity of the invariant sets for CDI systems.

Corollary 1. Let Assumptions 1 and 3 hold for the set-valued map $\mathcal{F}(\cdot)$ determining the system (1). Given a compact invariant set $\Omega \subseteq X$ with $0 \in \operatorname{int}(\operatorname{co}(\Omega))$, for the system (1), the set $\bar{\Omega}=\operatorname{co}(\Omega)$ is a convex, compact invariant set.

Proof: The proof is analogous to that one of Proposition 6.

Corollary 1 implies that the maximal invariant set in $X \subseteq \mathbb{R}^{n}$ is convex.

Corollary 2. Let Assumptions 1 and 3 hold for the set-valued map $\mathcal{F}(\cdot)$ determining the system (1) and the state constraint set $X \subseteq \mathbb{R}^{n}$. The maximal invariant set $\Omega_{M} \subseteq X$ is convex. 


\subsection{Robust invariance for uncertain CDI systems}

The results presented in the previous section can be extended to the CDI systems of the form (3). Given $\mathcal{F}(\cdot)$ in (3), define the set-valued function

$$
\mathcal{F}_{W}(x)=\left\{z \in \mathbb{R}^{n}: z \in \mathcal{F}(x) \oplus W\right\} .
$$

A characterization of contractiveness for the uncertain CDI systems is provided.

Proposition 7. Let Assumptions 1, 2 and 3 hold for the set-valued map $\mathcal{F}(\cdot)$ and the set $W$ determining the uncertain CDI system (3) and the state constraint set $X$. Given $\lambda \in[0,1]$, a set $\Omega \in \mathcal{K}^{0}(X)$ is a robust contractive set for system (3) if and only if

$$
\eta^{T} z \leq \lambda \phi_{\Omega}(\eta)-\phi_{W}(\eta), \quad \forall z \in \mathcal{F}(x), \quad \forall x \in \Omega, \quad \forall \eta \in \mathbb{R}^{n} .
$$

Proof: From properties of support functions we have that $\mathcal{F}(x) \oplus W \subseteq \lambda \Omega$, for all $x \in \Omega$, condition for robust contractiveness of $\Omega$, is equivalent to (18).

A boundary-type necessary and sufficient condition robust contractiveness follows. The proof is avoided since analogous to that one of Theorem 1.

Corollary 3. Let Assumptions 1, 2 and 3 hold for the set-valued map $\mathcal{F}(\cdot)$ and the set $W$ determining the uncertain CDI system (3) and the state constraint set $X$. Given $\lambda \in[0,1]$, a set $\Omega \in \mathcal{K}^{0}(X)$ is a robust contractive set for system (3) if and only if

$$
\check{F}(x, \eta) \leq \lambda \phi_{\Omega}(\eta)-\phi_{W}(\eta), \quad \forall x \in \partial \Omega, \quad \forall \eta \in \mathbb{R}^{n}
$$

\section{Computation of a contractive polytope for CDI systems}

Necessary and sufficient conditions stated in Theorems 1 and Corollary 3 are boundary-type ones. However, checking such conditions is not computationally affordable for generic $\Omega \in \mathcal{K}^{0}\left(\mathbb{R}^{n}\right)$, as they involve an infinite number of constraints, one for every $x \in \partial \Omega$ and for every $\eta \in \mathbb{R}^{n}$. On the contrary, for polytopic $\Omega \in \mathcal{K}^{0}\left(\mathbb{R}^{n}\right)$, defined as $\Omega=\left\{x \in \mathbb{R}^{n}: H x \leq 1\right\}$, with $H \in \mathbb{R}^{n_{h} \times n}$, the number of constraints is equal to $n_{v} n_{h}$, where $n_{v}$ is the numbers of vertices of $\Omega$.

Proposition 8. Let Assumptions 1 and 3 hold for the set-valued map $\mathcal{F}(\cdot)$ determining the system dynamics (1) and the state constraint set $X$. A polytope $\Omega=\left\{x \in \mathbb{R}^{n}: H x \leq 1\right\}$, with $H \in \mathbb{R}^{n_{h} \times n}$ and whose vertices are $v^{j} \in \mathbb{R}^{n}$ for $j \in \mathbb{N}_{n_{v}}$, is a contractive set with $\lambda \in[0,1]$ if and only if $\Omega \subseteq X$ and

$$
\check{F}\left(v^{j}, H_{i}^{T}\right) \leq \lambda, \quad \forall j \in \mathbb{N}_{n_{v}}, \quad \forall i \in \mathbb{N}_{n_{h}} .
$$


Proof: Since (14) is a necessary and sufficient condition for a generic $\Omega \in$ $\mathcal{K}^{0}(X)$ to be a contractive set for a CDI system, then the equivalence between (14) and (20) proves the proposition. From properties of support functions, condition (14) for polytopic $\Omega$ is given by

$$
\check{F}\left(x, H_{i}^{T}\right) \leq \lambda, \quad \forall x \in \partial \Omega, \quad \forall i \in \mathbb{N}_{n_{h}} .
$$

Moreover, from convexity of $\check{F}(\cdot, \eta)$, for all $\eta \in \mathbb{R}^{n}$, condition (21), involving $x \in \partial \Omega$, holds if and only if (20), concerning the vertices of $\Omega$, is satisfied.

Proposition 8 provides a necessary and sufficient condition for a polytope to be a contractive set for CDI systems, consisting of $n_{v} n_{h}$ convex constraints. The following result is useful to obtain a contractive set $\hat{\Omega}=\operatorname{co}(\Omega \cup\{\hat{x}\})$ by computing $\hat{x} \in X$, provided that $\Omega$ is a contractive polytope. Then $\Omega \subseteq \hat{\Omega}$ and the result permits to design an enlarging method for a contractive polytope.

Proposition 9. Let Assumptions 1 and 3 hold. Consider a polytope $\Omega=\{x \in$ $\left.\mathbb{R}^{n}: H x \leq 1\right\} \subseteq X$, with $H \in \mathbb{R}^{n_{h} \times n}$, and $\lambda \in[0,1]$, such that the hypothesis of Proposition 8 holds for $\Omega$, and, given $\hat{x} \in X$, define the set $\hat{\Omega}=\operatorname{co}(\Omega \cup\{\hat{x}\})$. If $\hat{x} \in X$ is such that $\check{F}\left(\hat{x}, H_{i}^{T}\right) \leq \lambda$, for every $i \in \mathbb{N}_{n_{h}}$, then $\hat{\Omega}$ is a contractive set for system (1) and the constraints $x \in X$.

Proof: From Proposition 8 we have that $\check{F}\left(x, H_{i}^{T}\right) \leq \lambda$ for all $i \in \mathbb{N}_{n_{h}}$, either if $x=\hat{x}$ or if $x$ is a vertex of $\Omega$. This implies, from convexity of $\check{F}(\cdot, \eta)$ for every $\eta \in \mathbb{R}^{n}$, that

$$
\check{F}\left(x, H_{i}^{T}\right) \leq \lambda, \quad \forall i \in \mathbb{N}_{n_{h}},
$$

for all $x \in \operatorname{co}(\Omega \cup\{\hat{x}\})=\hat{\Omega}$. Condition (22) is equivalent to $\mathcal{F}(x) \subseteq \lambda \Omega$, then, for every $x \in \hat{\Omega}$ we have that $\mathcal{F}(x) \subseteq \lambda \Omega \subseteq \lambda \hat{\Omega}$ which means that $\hat{\Omega}$ is a contractive polytope for the system (1).

The results stated in Propositions 8 and 9 are extended to the case of uncertain CDI systems.

Corollary 4. Let Assumptions 1, 2 and 3 hold for the set-valued map $\mathcal{F}(\cdot)$ and the set $W$ determining the uncertain CDI system (3) and the state constraint set $X$. A polytope $\Omega=\left\{x \in \mathbb{R}^{n}: H x \leq 1\right\}$, with $H \in \mathbb{R}^{n_{h} \times n}$ and whose vertices are $v^{j} \in \mathbb{R}^{n}$ for $j \in \mathbb{N}_{n_{v}}$, is a robust contractive set with $\lambda \in[0,1]$ if and only if $\Omega \subseteq X$ and

$$
\check{F}\left(v^{j}, H_{i}^{T}\right) \leq \lambda-\phi_{W}\left(H_{i}^{T}\right), \quad \forall j \in \mathbb{N}_{n_{v}}, \quad \forall i \in \mathbb{N}_{n_{h}} .
$$

Moreover, if $\hat{x} \in X$ is such that $\check{F}\left(\hat{x}, H_{i}^{T}\right) \leq \lambda-\phi_{W}\left(H_{i}^{T}\right)$, for every $i \in \mathbb{N}_{n_{h}}$, then $\hat{\Omega}=\operatorname{co}(\Omega \cup\{\hat{x}\})$ is a robust contractive set for system (3). 


\subsection{Algorithm}

The proposed algorithm provides a sequence of polytopic robust contractive sets for an uncertain CDI system with contracting factor $\lambda$. Assume that $\Omega_{L}=$ $\left\{x \in \mathbb{R}^{n}: H x \leq 1\right\}$, with $H \in \mathbb{R}^{n_{h} \times n}$, is an initial guess and $v^{j}$ are its $n_{v}$ vertices. A possibility to obtain the initial guess is to compute a contractive set for a system which is a local approximation, possibly linear, of the CDI one. Given a contractive set $\Omega$ for a linear approximation of the CDI (or nonlinear) system, there exists $\beta>0$ such that $\Omega_{L}=\beta \Omega$ is contractive for the CDI one, under certain differentiability assumptions (see [17] for an analogous result). Standard algorithms can be employed, see for instance $[3,5]$ and [7], to obtain $\Omega$. Alternatively, an LDI system, local extension of the CDI one, can be computed. Every contractive set for the LDI system is contractive also for the CDI one.

Algorithm 1. Computing a robust contractive set for a CDI system (3).

Given the CDI system (3) under Assumptions 1, 2 and 3 and the polytope $\Omega_{L}$ :

Solve:

$$
\alpha=\max _{\gamma>0} \gamma
$$

$$
\text { s.t. } \check{F}\left(\gamma v^{j}, H_{i}^{T}\right) \leq \lambda \gamma-\phi_{W}\left(H_{i}^{T}\right), \quad \forall j \in \mathbb{N}_{n_{v}}, \forall i \in \mathbb{N}_{n_{h}} \text {. }
$$

Pose $\Omega_{0}=\alpha \Omega_{L}=\left\{x \in \mathbb{R}^{n}: H^{0} x \leq 1\right\}$ and $k=0$.

for $k=0, \cdots, k_{\text {max }}$, randomly generate $\eta^{k} \in \mathbb{R}^{n}$ and solve:

$$
\begin{gathered}
x^{k}=\arg \max _{\hat{x} \in X}\left(\eta^{k}\right)^{T} \hat{x}, \\
\text { s.t. } \check{F}\left(\hat{x},\left(H_{i}^{k}\right)^{T}\right) \leq \lambda-\phi_{W}\left(\left(H_{i}^{k}\right)^{T}\right), \quad \forall i \in \mathbb{N}_{n_{h}^{k}}, \\
\text { and pose } \Omega_{k+1}=\operatorname{co}\left(\Omega_{k} \cup\left\{x^{k}\right\}\right)=\left\{x \in \mathbb{R}^{n}: H^{k+1} x \leq 1\right\} .
\end{gathered}
$$

end

The algorithm is based on Corollary 4. Given $\Omega_{L}$, the first step consists of computing the maximal $\alpha>0$ such that $\alpha \Omega_{L}$ is contractive for the CDI system. In fact $\gamma v^{j}$, with $j \in \mathbb{N}_{n_{v}}$, are the vertices of $\gamma \Omega_{L}$ and then the condition (24) implies that $\gamma \Omega_{L}$ is contractive. The following iteration generates a sequence of nested contractive sets, i.e. $\Omega_{k} \subseteq \Omega_{k+1}$, for every selection criterion of $\eta^{k} \in \mathbb{R}^{n}$. In fact $x^{k}$ is such that either $x^{k} \in \partial \Omega_{k}$ or $x^{k} \notin \Omega_{k}$ and satisfies the conditions 
of Corollary 4. Nevertheless it is desirable, in practice, to have a sequence converging to the maximal contractive set. Consider, with no loss of generality, the directions generated on the surface of the unitary ball in $\mathbb{R}^{n}$, denoted $\mathbf{B}^{n}$, and define

$$
N(\bar{\eta}, r)=\left\{\eta \in \partial \mathbf{B}^{n}:\|\bar{\eta}-\eta\|_{2}<r\right\},
$$

for every $\bar{\eta} \in \partial \mathbf{B}^{n}$ and $r>0$. That is, $N(\bar{\eta}, r)$ are the non-empty intersections of $\partial \mathbf{B}^{n}$ and open balls in $\mathbb{R}^{n}$.

Proposition 10. If the randomly generated directions $\eta^{k} \in \partial \mathbf{B}^{n}$ in Algorithm 1 are such that the probability of $\eta^{k} \in N(\bar{\eta}, r)$ is positive for every $\bar{\eta} \in \partial \mathbf{B}^{n}$ and $r>0$, then the sequence $\Omega_{k}$, with $k \in \mathbb{N}$, convergences to the maximal convex contractive set in $X$ for the CDI system (3).

Proof: Suppose that for $\eta^{k} \in \partial \mathbf{B}^{n}$ we have $x^{k} \notin \Omega$. This implies the existence of $\bar{\eta} \in \partial \mathbf{B}^{n}$ such that $\bar{\eta}^{T} x^{k}>\Phi_{\bar{\eta}}(\Omega)$, because of the separation theorem. From continuity of the support function with respect to $\eta$, for every bounded $\Omega$, we have that $f(\eta)=\eta^{T} x^{k}-\Phi_{\eta}(\Omega)$ is continuous and positive in $\bar{\eta} \in \partial \mathbf{B}^{n}$. Then, there exists a neighborhood of $\bar{\eta}$ such that $f(\eta)$ is positive for every $\eta$ in such a neighborhood. Thus, if there is $x^{k} \notin \Omega$ satisfying (25), which implies $\mathcal{F}\left(x^{k}\right) \oplus W \subseteq \Omega$, then there is $\bar{\eta} \in \partial \mathbf{B}^{n}$ and $r>0$ such that

$$
\max _{\hat{x} \in X}\left\{\eta^{T} \hat{x}: \text { s.t. }(25)\right\} \geq \eta^{T} x^{k}>\Phi_{\eta}(\Omega),
$$

for all $\eta \in N(\bar{\eta}, r)$. Thus if $\Omega_{k}$ can be enlarged, an enlarging direction will be found with non-zero probability. This implies that the sequence of nested contractive sets converges to the maximal one.

From Proposition 10, every criterion which selects a direction in any nonempty set $N(\bar{\eta}, r)$ with non-zero probability satisfies the requirements for convergence of $\Omega_{k}$ to the maximal convex contractive set. A possible choice is the uniform distribution.

Remark 6. Concerning the computational complexity of the algorithm, the first step is efficiently solvable, consisting of a convex optimization problem in the variable $\gamma \in \mathbb{R}$. This implies that the computation of the contractive polytope $\Omega_{0}$ can be performed for high dimensional problems, provided that $\Omega_{L}$ and its vertices are known. The iteration concerning the enlarging procedure, on the other hand, requires a high computational burden. In fact, although (25) is a convex optimization problem in the variable $\hat{x}$, the computation of $\Omega_{k+1}$ consists of a convex hull 
operation and a simplification process to generate the minimal $H$-representation of the polytope. Both these two sub-procedures are computationally demanding. Then the enlarging procedure should be performed only for relatively low dimensional problems, as illustrated in the following example.

Example 1. To give an idea of which is the largest dimension for the problem to be solved in reasonable time, we applied Algorithm 1 to a generalized saturated system varying its dimension. For $n=6$, the Matlab procedure requires some minutes to compute the sequence of $\Omega_{k}$ for $k_{\max }=9$, with a non-optimized code and using standard Matlab routines (for polytopes handling, for instance). Table 1 shows the evolution of the number of vertices and facets of $\Omega_{k}$ for $n=6$. The increase of the number of vertices and facets yields the enlarging procedure to be more and more time-consuming as the algorithm proceeds.

\begin{tabular}{|c|cccccccccc|}
\hline Step & 0 & 1 & 2 & 3 & 4 & 5 & 6 & 7 & 8 & 9 \\
\hline Vertices & 65 & 66 & 67 & 68 & 69 & 70 & 71 & 72 & 73 & 74 \\
\hline Facets & 28 & 44 & 59 & 75 & 105 & 128 & 164 & 191 & 246 & 300 \\
\hline
\end{tabular}

Table 1: Vertices and facets of $\Omega_{k} \subseteq \mathbb{R}^{n}$, with $n=6$.

Then, for relatively low dimensional systems, the choice of the particular geometry of the initial guess $\Omega_{L}$ is not crucial, as the enlarging process permits to generate properly shaped contractive sets. On the contrary, the selection of $\Omega_{L}$ can strongly influence the size of the contractive sets obtained for higher dimensional systems, as the enlarging iterations can result computationally unaffordable. On the other hand, the problem of computing contractive sets for nonlinear systems is rather complex by its nature. Hence, a trade-off between the size of the obtained contractive sets and the required computational complexity is unavoidable, in our opinion, for high dimensional systems.

\section{Numerical example}

Consider a generalized saturated system (9), see Section 2.2, with matrices

$$
A=\left[\begin{array}{cc}
1.1 & 1 \\
0 & 1.1
\end{array}\right], \quad B=\left[\begin{array}{l}
0.5 \\
1.1
\end{array}\right], \quad F=\left[\begin{array}{ll}
-0.5236 & -1.1264
\end{array}\right]
$$

and $\Gamma(y)=\max \left\{\mu(y+\sigma),-y_{0}\right\}$ with $\mu=1, \sigma=0.2$ and $y_{0}=1.8$, as in Definition 2. The CDI extension of the generalized saturated system is determined by 
convex bounding functions given by (10), with $\Gamma^{0}(y)=\max \left\{\mu y,-y_{0}-\mu \sigma\right\}=$ $\max \{y,-2\}$, and by $W=\{w=B v:-0.2 \leq v \leq 0.2\}$, see Section 2.2. The state is assumed to be constrained in the region $X=\left\{x \in \mathbb{R}^{2}:-15 \leq\right.$ $\left.x_{1} \leq 15,-6 \leq x_{2} \leq 6\right\}$. Notice that in the region of the state space given by $D=\left\{x \in \mathbb{R}^{n}:|F x| \leq \frac{y_{0}}{\mu}+\sigma\right\}=\left\{x \in \mathbb{R}^{n}:|F x| \leq 2\right\}$, the CDI system is equal to the linear one given by $x_{k+1}=(A+B F) x_{k}+w_{k}$, whose eigenvalues are $0.3496 \pm 0.1133 i$, lying in the unitary circle. Such linear system is used to determine a local invariant set $\Omega_{L}$ using standard iterative methods. Since we are interested in a robust invariant set for the uncertain CDI system, we choose $\lambda=1$ and apply the algorithm.

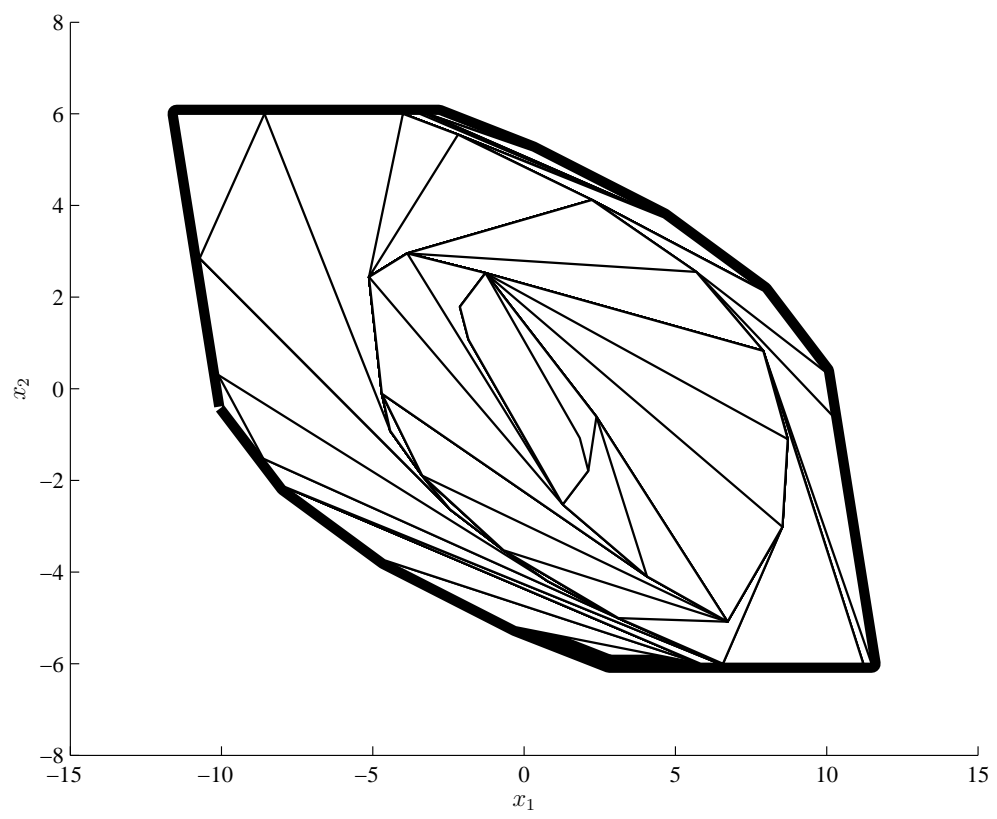

Figure 2: Sequence of sets $\Omega_{k}$ for the CDI system, for $k \in \mathbb{N}_{\left[0, k_{\text {max }}\right]}$, generated by Algorithm 1.

In Figure 2, the sequence of robust invariant sets generated by the enlarging process are depicted. The inner set is $\Omega_{0}=\alpha \Omega_{L}$ computed at first step of the algorithm. The biggest robust invariant set is $\Omega_{k_{\max }}$, with $k_{\max }=100$. Notice that the state constraints are satisfied, i.e. $\Omega_{k} \in X$.

\section{Conclusions}

In this paper the CDI modelling framework has been presented and used to characterize invariance and contractiveness of convex sets for nonlinear and un- 
certain systems. Conditions for invariance and contractiveness are posed as a set of constraints involving convex bounding functions. Thanks to the properties of convexity, such constraints are boundary-type conditions, unlike the case of generic nonlinear systems. This led to the definition of a procedure for computing polytopic invariant sets based on convex constraints satisfaction for CDI systems.

One future research direction concerns further developments of the theoretical aspects of the CDI systems, considering for instance the problems of design and estimation. On the other hand, the particularization of the properties of the CDI systems to specific subclasses of nonlinear ones, saturated and generalized saturated for instance, could lead to extend and improve the results for common and more practice-oriented models.

\section{References}

[1] D. P. Bertsekas, Infinite-time reachability of state-space regions by using feedback control, IEEE Transactions on Automatic Control 17 (1972) 604613.

[2] P. Gutman, M. Cwikel, Admissible sets and feedback control for discretetime linear dynamical systems with bounded control and states, IEEE Transactions on Automatic Control AC-31 (4) (1986) 373-376.

[3] E. G. Gilbert, K. Tan, Linear systems with state and control constraints: The theory and application of maximal output admissible sets, IEEE Transactions on Automatic Control 36 (1991) 1008-1020.

[4] F. Blanchini, Ultimate boundedness control for discrete-time uncertain systems via set-induced Lyapunov functions, IEEE Transactions on Automatic Control 39 (1994) 428-433.

[5] I. Kolmanovsky, E. G. Gilbert, Theory and computation of disturbance invariant sets for discrete-time linear systems, Mathematical Problems in Engineering 4 (1998) 317-367.

[6] S. V. Raković, E. C. Kerrigan, K. I. Kouramas, D. Q. Mayne, Invariant approximations of the minimal robust positively invariant set, IEEE Transactions on Automatic Control 50 (2005) 406-410.

[7] F. Blanchini, S. Miani, Set-Theoretic Methods in Control, Birkhäuser, 2008. 
[8] D. Q. Mayne, J. B. Rawlings, C. V. Rao, P. O. M. Scokaert, Constrained model predictive control: Stability and optimality, Automatica 36 (2000) 789-814.

[9] E. F. Camacho, C. Bordóns, Model Predictive Control, Springer-Verlag, 2004.

[10] L. Magni, G. De Nicolao, L. Magnani, R. Scattolini, A stabilizing modelbased predictive control algorithm for nonlinear systems, Automatica 37 (2001) 1351-1362.

[11] M. Cannon, V. Deshmukh, B. Kouvaritakis, Nonlinear model predictive control with polytopic invariant sets, Automatica 39 (2003) 1487-1494.

[12] J. M. G. da Silva, S. Tarbouriech, Polyhedral regions of local stability for linear discrete-time systems with saturating controls, IEEE Transactions on Automatic Control 44 (1999) 2081-2085.

[13] T. Hu, Z. Lin, B. M. Chen, Analysis and design for discrete-time linear systems subject to actuator saturation, Systems \& Control Letters 45 (2) (2002) $97-112$.

[14] T. Alamo, A. Cepeda, D. Limón, E. F. Camacho, A new concept of invariance for saturated systems, Automatica 42 (2006) 1515-1521.

[15] T. Alamo, A. Cepeda, M. Fiacchini, E. F. Camacho, Convex invariant sets for discrete-time Lur'e systems, Automatica 45 (2009) 1066-1071.

[16] J. M. Bravo, D. Limón, T. Alamo, E. F. Camacho, On the computation of invariant sets for constrained nonlinear systems: An interval arithmetic approach, Automatica 41 (2005) 1583-1589.

[17] M. Fiacchini, T. Alamo, E. F. Camacho, On the computation of local invariant sets for nonlinear systems, in: Proceedings of 46th IEEE Conference on Decision and Control, CDC 2007, New Orleans, LA, USA, 2007.

[18] M. Fiacchini, T. Alamo, E. F. Camacho, On the computation of convex robust control invariant sets for nonlinear systems, Automatica 46 (8) (2010) 1334-1338. 
[19] H. Haimovich, E. Kofman, M. M. Seron, Systematic ultimate bound computation for sampled-data systems with quantization, Automatica 43 (6) (2007) $1117-1123$.

[20] J. Aubin, A. Cellina, Differential Inclusions: Set-Valued Maps and Viability Theory, Springer, 1984.

[21] J. Aubin, H. Frankowska, Set-valued analysis, Birkhäuser, 1990.

[22] J. Aubin, Viability theory, Birkhäuser, 1991.

[23] R. T. Rockafellar, Convex Analysis, Princeton University Press, USA, 1970.

[24] R. Schneider, Convex bodies: The Brunn-Minkowski theory, Cambridge University Press, 1993.

[25] S. Boyd, L. Vandenberghe, Convex Optimization, Cambridge University Press, 2004.

[26] M. Fiacchini, Convex difference inclusions for systems analysis and design, Ph.D. thesis, Universidad de Sevilla, Spain (January 2010).

[27] S. Boyd, L. El Ghaoui, E. Feron, V. Balakrishnan, Linear Matrix Inequalities in system and control theory, SIAM, 1994.

[28] L. Gurvits, Stability of discrete linear inclusion, Linear Algebra and its Applications 231 (1) (1995) $47-85$.

[29] M. V. Kothare, V. Balakrishnan, M. Morari, Robust constrained model predictive control using linear matrix inequalities, Automatica 32 (1996) 13611379.

[30] S. Tarbouriech, I. Queinnec, T. Alamo, M. Fiacchini, E. Camacho, Ultimate bounded stability and stabilization of linear systems interconnected with generalized saturated functions, Automatica 47 (7) (2011) 1473-1481. 